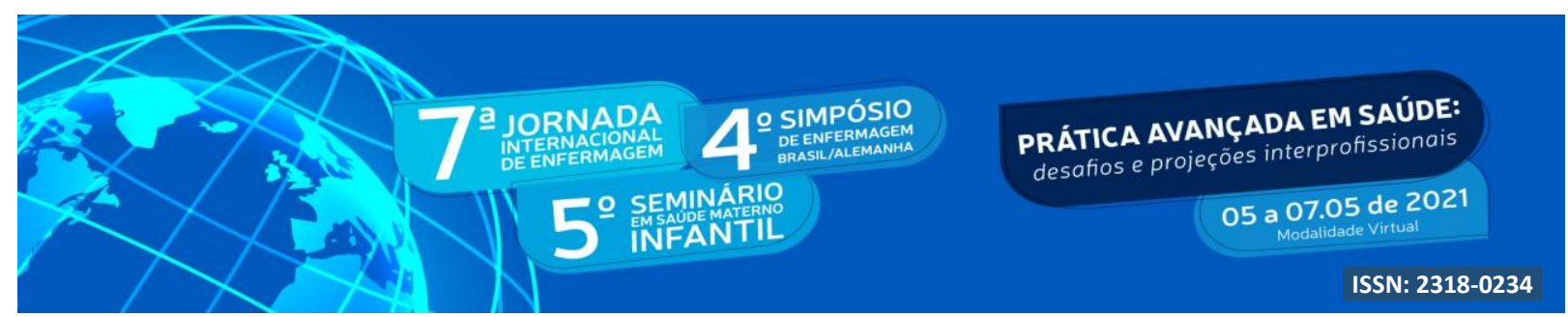

DOI: http://doi.org/10.48195/jie2021-101

\title{
CARACTERIZAÇÃO SOCIODEMOGRÁFICA DE CUIDADORES DE USUÁRIOS EM ATENÇÃO DOMICILIAR ${ }^{1}$
}

\section{Claudia Maria Ferrony Rivas²; Eduardo Marques Machado³; Natalia Cassol Bolzan; Clandio Timm Marques5; Maria Helena Gehlen'; Naiana Oliveira dos Santos?.}

\begin{abstract}
RESUMO
Este estudo objetivou descrever o perfil sociodemográfico dos cuidadores de usuários cadastrados em um serviço de Atenção Domiciliar do município de Santa Maria/RS. Estudo transversal e quantitativo, desenvolvido em uma equipe da Estratégia Saúde da Família da zona norte do município de Santa Maria/RS. Para a coleta de dados sociodemográficos foi utilizado um instrumento desenvolvido pelos pesquisadores. A amostra foi constituída por 51 cuidadores. Evidenciou-se a prevalência de cuidadores primários, do sexo feminino, filhas/filhos do usuário, com idade média de 49,1 $\pm 17,3$ anos, casadas(os) e com baixa escolaridade (ensino fundamental incompleto/completo). Conclui-se assim que se torna imprescindível para um cuidado efetivo e humanizado no ambiente domiciliar o reconhecimento da importância do papel do cuidador na atenção à saúde ao usuário, sendo fundamental que os profissionais elaborarem o planejamento da assistência em saúde atentando para a figura do cuidador no processo de cuidar.
\end{abstract}

Palavras-chave: Cuidadores; Enfermagem; Assistência Domiciliar.

\begin{abstract}
This study aimed to describe the sociodemographic profile of caregivers of users registered in a Home Care service in the city of Santa Maria/RS. Cross-sectional and quantitative study developed in a team from the Family Health Strategy in the northern area of the municipality of Santa Maria/RS. For the collection of sociodemographic data, an instrument developed by the researchers was used. The sample consisted of 51 caregivers. The prevalence of primary caregivers, female, daughters/sons of the user, with an average age of $49.1 \pm 17.3$ years, married and with low education (incomplete/complete elementary school) was evidenced. It is concluded, therefore, that it is essential for effective and humanized care in the home environment to recognize the importance of the role of the caregiver in health care for the user, and it is essential that professionals prepare the planning of health care, paying attention to the figure of the caregiver in the care process.
\end{abstract}

Key Words: Caregivers; Nursing; Home Care

\footnotetext{
${ }^{1}$ Trabalho vinculado a bolsa de pesquisa de iniciação científica-FAPERGS.

${ }^{2}$ Estudante do Curso de Enfermagem. Bolsista de iniciação científica-FAPERGS. Universidade FranciscanaUFN. E-mail: claudiamfrivas@gmail.com

${ }^{3}$ Estudante do Curso de Enfermagem. Universidade Franciscana-UFN.E-mail: eduardomachado051@ gmail.com

${ }^{4}$ Estudante do Curso de Enfermagem. Universidade Franciscana-UFN. E-mail: natalia.bolzan23@gmail.com

${ }^{5}$ Professor do Curso de Matemática -Universidade Franciscana-UFN. E-mail: clandio@ufn.edu.br

${ }^{6}$ Professora do Curso de Enfermagem-Universidade Franciscana. E-mail: gehlenmh@gmail.com

${ }^{7}$ Orientadora e pesquisadora responsável pela pesquisa. Professora do curso de Enfermagem-Universidade Franciscana-UFN. E-mail: naiaoliveira07@gmail.com
} 


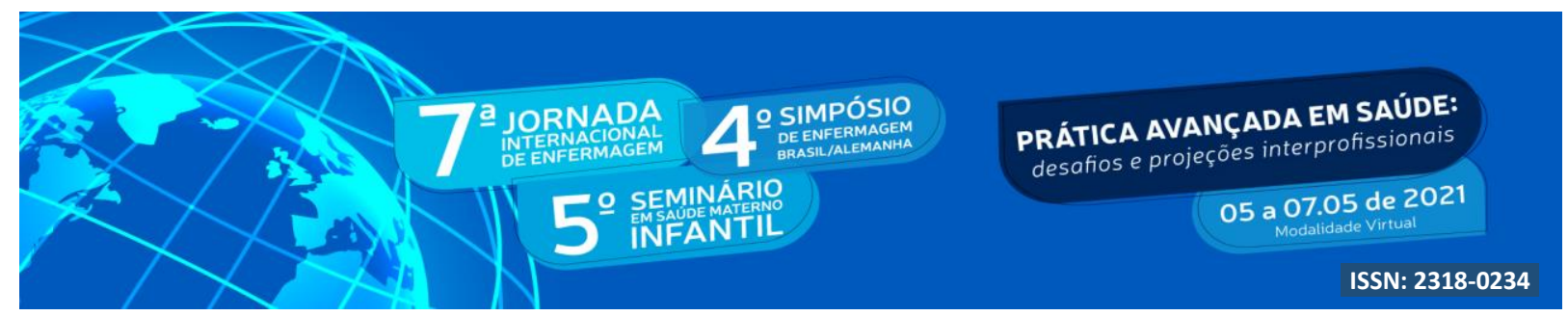

\section{INTRODUÇÃO}

Mudanças das necessidades de saúde nas diferentes faixas etárias da população brasileira são constatadas ao longo dos anos. Essas transformações, exigem respostas do sistema de saúde de acordo com as demandas apresentadas. Com o aumento considerável de atendimentos a população idosa, a presença de usuários com doenças crônicas degenerativas ou com complicações oriundas de acidentes ou doenças e o entrave ao acesso aos serviços de saúde, a Atenção Domiciliar (AD) destaca-se como uma relevante possibilidade de resposta do Sistema Único de Saúde (SUS) (BRASIL, 2020).

A AD é definida por ser uma modalidade de atenção à saúde realizada no domicílio, com caráter substitutivo ou complementar aos demais serviços de saúde. Está integrada a Rede de Atenção à Saúde (RAS) e é pautada em uma série de ações para prevenção, paliação, tratamento de doenças, reabilitação e promoção da saúde assegurando assim a continuidade de cuidados (BRASIL, 2016).

No ano 2011, houve a expansão da AD no país, por meio da Portaria $n^{\circ} 2029$, que instituiu a Política Nacional de Atenção Domiciliar e a criação do Programa Melhor em Casa (PMC). De acordo com a Política, o AD é indicada para os usuários que estão estáveis clinicamente e que estejam restritos ao leito ou ao domicílio, de forma temporária ou definitiva ou em condições de vulnerabilidade (BRASIL, 2011).

Organiza-se a AD em três modalidades: AD1 para os usuários que necessitam menor frequência de cuidado e menor acompanhamento profissional, sobre responsabilidade da Atenção Primária de Saúde (APS); AD2, para usuários que requeiram mais recursos e cuidados da equipe multiprofissional, e AD3 para usuários que precisem tecnologias de cuidado mais complexas. As duas últimas modalidades estão sobre responsabilidade do Serviço de Atenção Domiciliar (SAD) (BRASIL, 2016).

Observa-se diferentes desafios para a consolidação do cuidado no domicílio, tais como a elaboração do plano de cuidados, a competência dos profissionais em saúde para a prestar o cuidado de modo integral e em equipe, estando no ambiente íntimo do usuário (RAJÃO e MARTINS, 2019).

Neste contexto do trabalho em equipe no domicílio, destaca-se o papel do cuidador, 


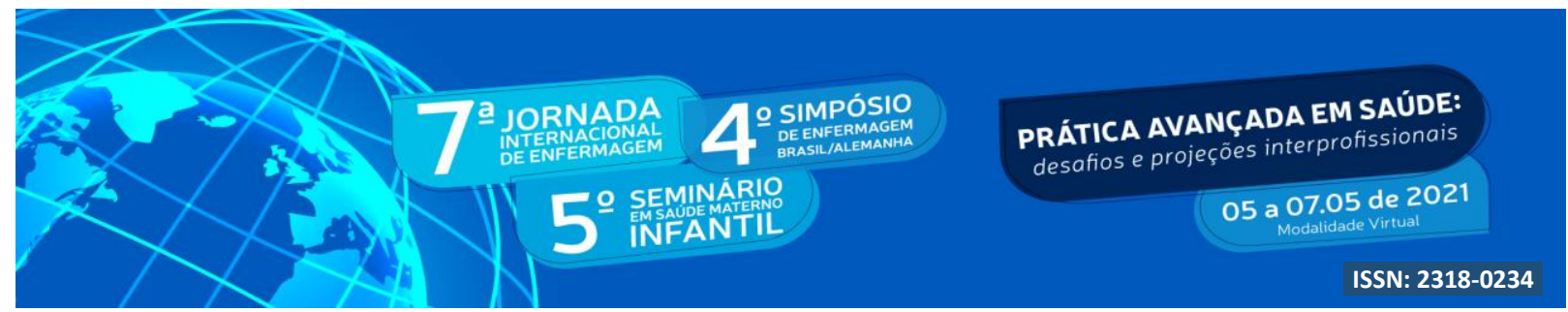

sujeito com ou sem vínculo familiar, que de acordo com as condições funcionais e clínicas do usuário, deverá estar presente e capacitado para ajudar nas demandas do usuário e em suas atividades rotineiras (BRASIL, 2016).

Os cuidadores no contexto da $\mathrm{AD}$, são geralmente cuidadores informais, ou seja, familiares ou pessoas próximas ao usuário que assumem a responsabilidade pelo cuidado no domicílio, divergindo dos cuidadores informais, indivíduos que em sua maioria tem formação específica para desempenhar a função de cuidar e são remunerados para o propósito (FALEIROS et al, 2015).

Neste sentido é imprescindível conhecer a realidade dos cuidadores, já que estes vivenciam além das situações benéficas, experiências que podem gerar sobrecarga física e mental, carência de apoio e insegurança, necessitando dos profissionais da saúde, uma visão mais singular ao organizar o cuidado na $\mathrm{AD}$, que contemple dar visibilidade aos diversos atores envolvidos no cuidado (PROCÓPIO et al, 2019).

\section{OBJETIVO}

Descrever o perfil sociodemográfico dos cuidadores de usuários cadastrados em um serviço de AD do município de Santa Maria/RS.

\section{METODOLOGIA}

Trata-se de um estudo transversal e quantitativo, desenvolvido no contexto de uma equipe da Estratégia Saúde da Família da zona norte do município de Santa Maria/RS. Foram incluídos na pesquisa todos os cuidadores de usuários cadastrados ao AD das ESF no período do estudo, de setembro a dezembro de 2019. Foram excluídos da pesquisa os cuidadores de usuários não contactados pelos pesquisadores, após três tentativas em dias e turnos diferentes.

Para a coleta de dados sociodemográficos foi utilizado um instrumento de coleta de dados desenvolvida pelos pesquisadores, composto pelas seguintes informações: sexo (feminino e masculino); idade; grau de parentesco (filha/filho, cônjuge, irmã/irmão, nora/genro, neto, pai/mãe e outros); estado civil (solteiro, casado, viúvo, divorciado); escolaridade (analfabeto, ensino fundamental incompleto/completo, ensino médio 


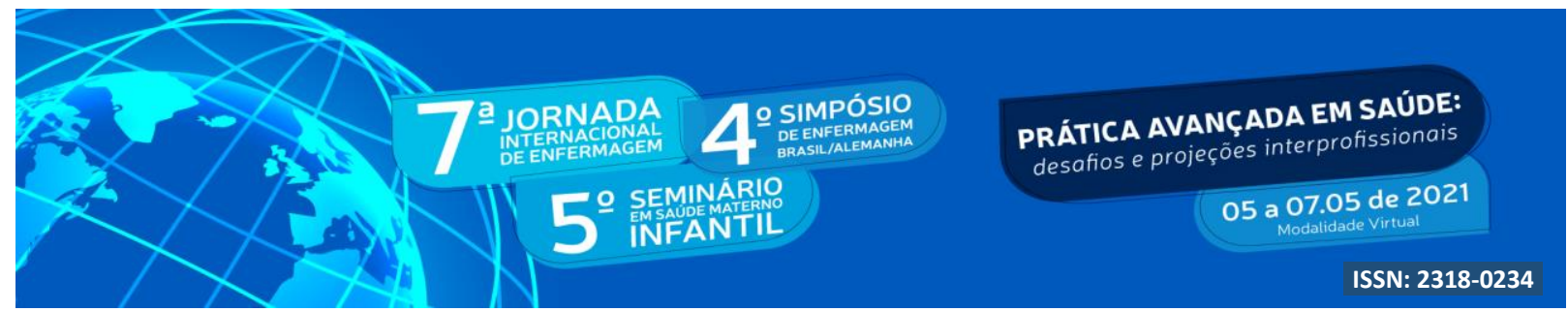

incompleto/completo; superior incompleto/completo). Atendendo os objetivos de caracterização dos cuidadores utilizou-se a estatística descritiva.

A pesquisa foi aprovada pelo Comitê de Ética em Pesquisa da Universidade Franciscana, sob o CAEE: 11715019.6.0000.530. O estudo respeitou todos os preceitos éticos do Conselho Nacional de Saúde, que dispõe sobre as diretrizes e normas regulamentadoras da pesquisa envolvendo seres humanos

\section{RESULTADOS E DISCUSSÃO}

A amostra final foi constituída por 51 cuidadores de usuários em AD. Em sua maioria os cuidadores eram do sexo feminino $(n=40)$. Os dados corroboram com outros estudos que demonstram a prevalência de cuidadores do sexo feminino (ROSAS e NERI, 2019; MAMAMI et al, 2019; ALMEIDA et al, 2018; SANTOS e KOETZ, 2017). Percebe-se que geralmente as mulheres acabam por desempenhar o ato de cuidar, resultado de um contexto histórico, social e cultural que condiciona a mulher a exercer o papel social de prover o cuidado da casa e da família (FERREIRA; ISAAC e XIMENES, 2018; ALMEIDA et al, 2018; BRASIL, 2013).

Nesta perspectiva observa-se a importância de desenvolver políticas sociais e de saúde as mulheres e de buscar por transformações relacionadas a encarregar ao familiar mais apto a execução do cuidado, dissociando o gênero como determinante para o desempenho da função (FERREIRA; ISAAC e XIMENES, 2018).

A idade mínima dos cuidadores variou de 19 anos a máxima de 90 anos, com média de 49,1 \pm 17,3 anos. Foi observado que 43 cuidadores eram informais e oito cuidadores eram contratados. No que se refere ao grau de parentesco, 19 cuidadores eram filhas/filhos; nove eram cônjuges; quatro tinham outro tipo de parentesco, um era irmão; uma era nora/genro; um neto(a); dez cuidadores eram pai/mãe e seis não possuíam vínculo familiar.

Os dados da pesquisa refletem que a maioria dos cuidadores eram do círculo familiar do usuário. Estudos semelhantes apontam majoritariamente filhas/filhas como cuidadores, seguido de cônjuges (SANTOS e KOETZ, 2017; MAMAMI et al, 2019; LOPES et al, 2020). Outros estudos divergem dos dados da pesquisa, mostrando os cônjuges como os principais responsáveis pelo cuidado seguido de filha/filho (ALMEIDA et al, 2018; ROSAS e NERI, 


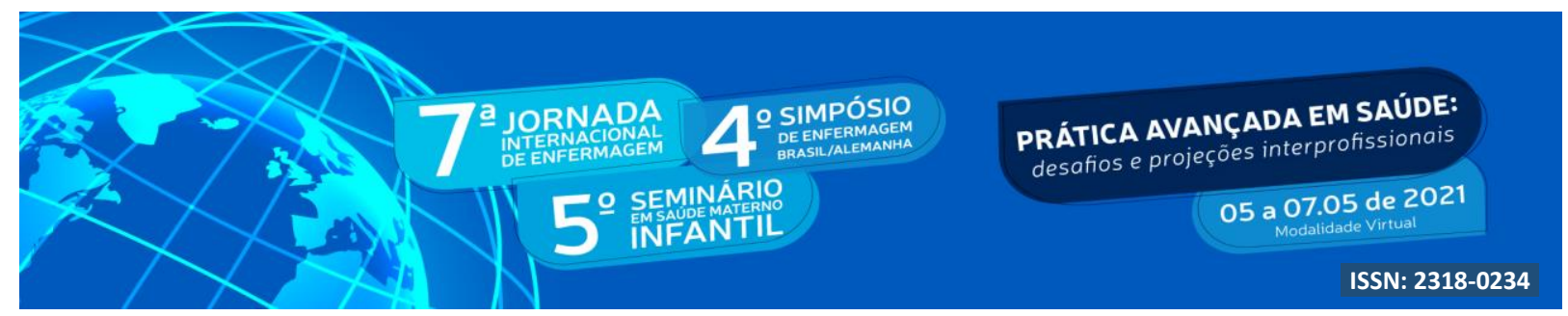

2019).

Compreende-se que ser do círculo familiar influencia a designação do cuidador. E que, ao ter esse vínculo, muitos familiares reconhecem como obrigatoriedade moral o cuidar, exercendo os laços familiares importante aspecto para o processo de torna-se um cuidador (FERREIRA; ISAAC e XIMENES, 2018; SANTOS e KOETZ, 2017).

Identificou-se no que concerne ao estado civil que 30 usuários eram casados; 12 usuários solteiros; sete usuários divorciados e dois viúvos. Pesquisas ratificam esses dados já constatam que a maioria dos cuidadores são casados (ALMEIDA et al, 2018, MAMAMI et al, 2019; ALVES et al, 2019). Outro estudo que aborda sobre as situações vivenciadas por cuidadores familiares de idosos na $\mathrm{AD}$, equipara o número de cuidadores casados com solteiros (SILVA et al, 2019).

Em relação a escolaridade, 28 usuários possuíam o ensino fundamental incompleto/ completo, 19 cuidadores concluíram o ensino médio incompleto/completo, dois relataram não ter estudado, um cuidador não recordava o seu grau de escolaridade e apenas um cuidador havia estudado/concluído o ensino superior. Em consonância com esses dados, diversos estudos destacam a baixa escolaridade dos cuidadores, possuindo a maior parte dos cuidadores o ensino fundamental incompleto (ALMEIDA et al, 2018; SILVA et al, 2019; LOPES et al, 2020; ORLANDI et al, 2019).

Sabe-se que o nível instrução educacional pode interferir no conhecimento necessário para execução dos cuidados. A capacidade de aprendizado está intimamente atrelada a efetiva compreensão das ações de educação em saúde (ALMEIDA et al, 2018). Sendo assim, instruções claras, viáveis e objetivas, promovidas pelos profissionais aos cuidadores, devem ser inseridas na proposta de plano de cuidados aos usuários em $\mathrm{AD}$, permitindo que a educação em saúde seja alicerçada na aptidão do cuidador para realizá-lo.

\section{CONCLUSÃO}

O estudo descreveu o perfil sociodemográfico de cuidadores de usuários em AD vinculados a uma ESF do municipio de Santa Maria/Rs. Evidenciou-se a prevalência de cuidadores primários, do sexo feminino, filhas/filhos do usuário, com idade média de 49,1 \pm 17,3 anos, casadas(os) e com baixa escolaridade (ensino fundamental incompleto/completo). 


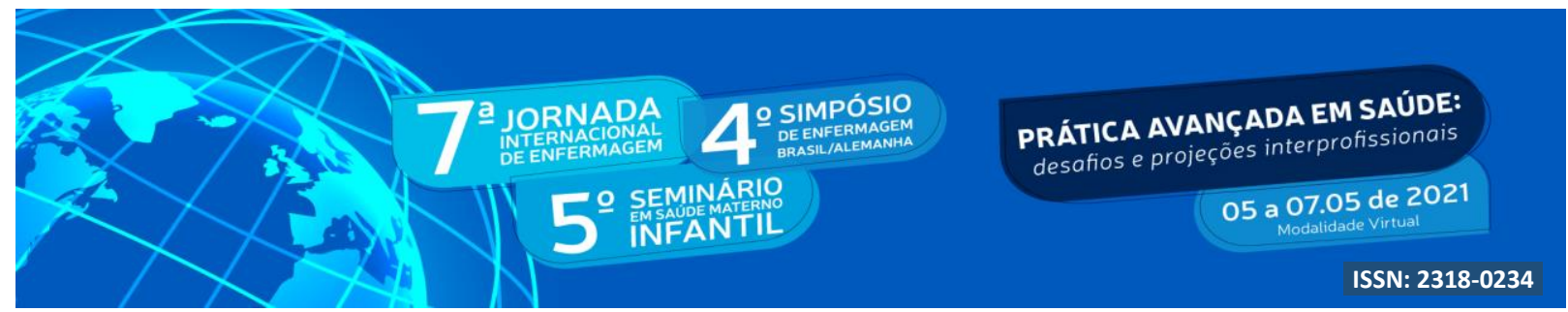

Conhecer as características sociodemográficas dos cuidadores de usuários em AD, possibilita compreender, informações importantes que podem subsidiar estratégias de educação em saúde junto aos cuidadores.. Dessa maneira, espera-se que o estudo possa contribuir para que os profissionais da saúde, possam planejar e implementar um plano de cuidados na $\mathrm{AD}$ mais personalizado, contemplando as características mais evidentes dos cuidadores.

No que se refere as limitações desse estudo, pode-se citar o tamanho pequeno da amostra em um determinado território.Conclui-se assim que torna-se imprescindível para um cuidado efetivo e humanizado no ambiente domiciliar e para o fortalecimento da AD como potencial estratégia de cuidado, o reconhecimento da importância do papel do cuidador na atenção a saúde ao usuário, sendo fundamental que os profissionais elaborarem o planejamento da assistência em saúde atentando para a figura do cuidador no processo de cuidar.

\section{REFERÊNCIAS}

ALMEIDA, L.de P.B., et al. Características sociais e demográficas de idosos cuidadores e motivos para cuidar da pessoa idosa em domicílio.REME • Rev Min Enferm.v.22, 2018. [Citado em 08 Março 2021]. Disponível em: https://cdn.publisher.gn1.link/reme.org.br/pdf/e1074.pdf

ALVES, B. S., et al. Caracterização dos cuidadores informais de idosos dependentes quanto aos aspectos demográficos e de saúde. Revista de Saúde Coletiva da UEFS, v. 9, p. 113118, 2019.[Citado em 10 Março 2021] . Disponível em: http://dx.doi.org/10.13102/rscdauefs.v9i0.368

BRASIL. Atenção domiciliar na atenção primária à saúde [recurso eletrônico] / Brasília : Ministério da Saúde, 2020. [Citado em 04 Março 2021]. Disponível em: https://bvsms.saude.gov.br/bvs/publicacoes/atencao_domiciliar_primaria_saude.pdf

BRASIL. Melhor em Casa. Caderno de atenção domiciliar, Brasilia: Ministério da Saúde, v.1, 2013. [Citado em 08 de Março 2021]. Disponível em:

https://bvsms.saude.gov.br/bvs/publicacoes/caderno_atencao_domiciliar_melhor_casa.pdf

BRASIL. Portaria $\mathrm{n}^{\circ}$ 825, de 25 de abril de 2016. Redefine a atenção domiciliar no âmbito do Sistema Único de Saúde (SUS) e atualiza as equipes habilitadas. Diário Oficial da União, Brasília: Ministério da Saúde, 2016.[Citado em 05 Março 2021]. Disponível em: https://bvsms.saude.gov.br/bvs/saudelegis/gm/2016/prt0825_25_04_2016.html 


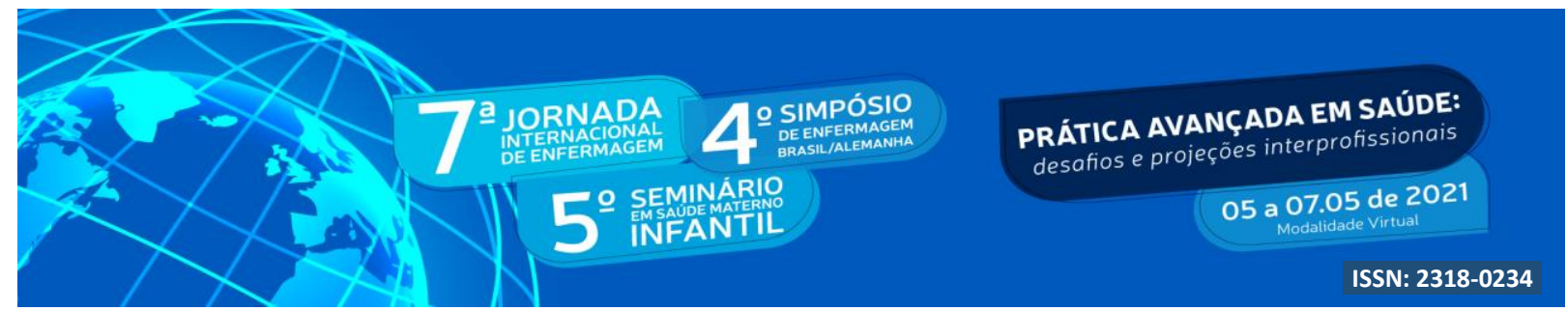

BRASIL. Portaria ${ }^{\circ} 2029$, de 24 de agosto de 2011. Institui a atenção domiciliar no âmbito do Sistema Único de Saúde (SUS), Brasília: Ministério da Saúde, 2011.[Citado em 05 março 2021]. Disponível em: http://bvsms.saude.gov.br/bvs/saudelegis/gm/2011/prt2029_24_08

FALEIROS, A. H., et al. Os Desafios do Cuidar: Revisão Bibliográfica, Sobrecargas e Satisfações do Cuidador de Idosos. Janus, Lorena, p. 59-68, 2015.[Citado em 16 Março 2021]. Disponível em: https://docplayer.com.br/54174752-Os-desafios-do-cuidar-revisaobibliografica-sobrecargas-e-satisfacoes-do-cuidador-de-idosos.html 18.03

FERREIRA, C. R.; ISAAC, L.; XIMENES, V. S. Cuidar de idosos: um assunto de mulher?. Est. Inter. Psicol., Londrina, v. 9, n. 1, p. 108-125, 2018.[Citado em 07 Março 2021]. Disponível em: http://pepsic.bvsalud.org/scielo.php?script=sci_arttext\&pid=S2236$\underline{64072018000100007 \& \operatorname{lng}=\mathrm{pt} \& \mathrm{nrm}=\mathrm{iso}}$

LOPES, C. C., et al . Associação entre a ocorrência de dor e sobrecarga em cuidadores principais e o nível de independência de idosos nas atividades de vida diária: estudo transversal. Cad. saúde colet., Rio de Janeiro, v. 28, n. 1, p. 98-106, 2020.[Citado em 10 Março 2021]. Disponível em: http://dx.doi.org/10.1590/1414-462x202028010184

MAMANI, A. R. N., et al . Cuidador de idosos: conhecimentos, atitudes e práticas sobre quedas e sua prevenção. Rev. Bras. Enferm., Brasília , v. 72, supl. 2, p. 119-126, 2019. [Citado em 07 Março 2021]. Disponível em: https://doi.org/10.1590/0034-7167-2018-0276.

ORLANDI, A. A. S., et al . Idosos cuidadores de idosos: fragilidade, solidão e sintomas depressivos. Rev. Bras. Enferm., Brasília, v. 72, supl. 2, p. 88-96, 2019.[Citado em 10 Março 2021]. Disponível em: http://dx.doi.org/10.1590/0034-7167-2018-0137

PROCOPIO, L. C. R.; et al . A atenção domiciliar no âmbito do Sistema Único de Saúde: desafios e potencialidades. Saúde debate, Rio de Janeiro , v. 43, n. 121, p. 592-604, 2019. [Citado em 05 Março 2021]. Disponível em: https://doi.org/10.1590/0103-1104201912123.

RAJAO, F. L.; MARTINS, M. Atenção domiciliar no Brasil: estudo exploratório sobre a consolidação e uso de serviços no Sistema Único de Saúde. Ciênc. saúde coletiva, Rio de Janeiro, v. 25, n. 5, p. 1863-1877, 2020.[Citado em 05 Março 2021]. Disponível em: https://doi.org/10.1590/1413-81232020255.34692019

ROSAS, C.; NERI, A. L. Qualidade de vida, sobrecarga, apoio emocional familiar: um modelo em idosos cuidadores. Rev. Bras. Enferm. , Brasília, v. 72, supl. 2, pág. 169-176, 2019.[Citado em 07 Março 2021]. Disponível em: https://doi.org/10.1590/0034-7167-2018$\underline{0439}$

SANTOS, B. E. , KOETZ, L. C. E. O perfil socioepidemiológico e a autopercepção dos cuidadores familiares sobre a relação interpessoal e o cuidado com idosos. Revista Acreditação: ACRED, v. 7, n. 13, 2017, p. 115-132 [Citado em 08 de março 2021]. Disponível em:

https://dialnet.unirioja.es/servlet/articulocodigo6130788:textCentro20UniversitC3A1rio20Uni 


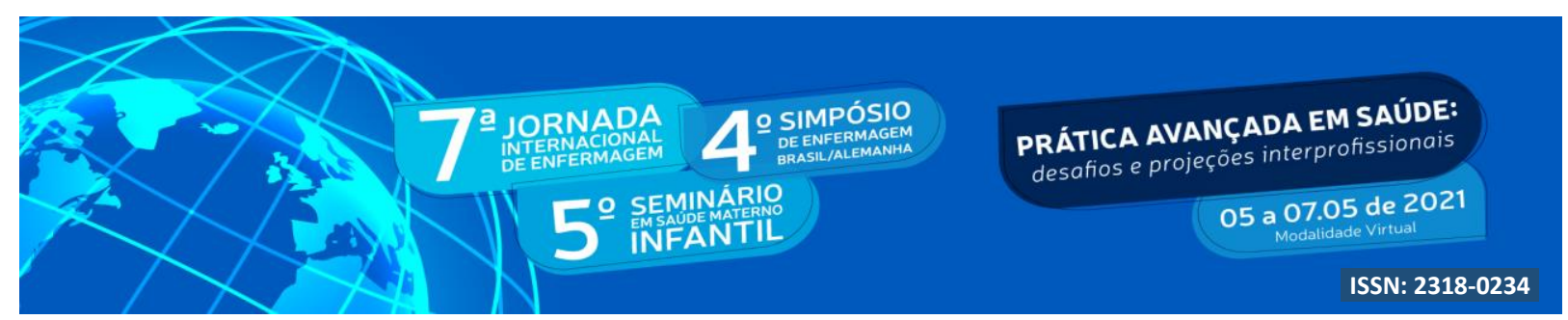

vates,Brasil\&textCuidar20de20um20idoso20dependente,serviC3A7os20direcionados20para2 0sua20saC3BAde.

SILVA M.S., et al. Situações vivenciadas por cuidadores familiares de idosos na atenção domiciliar. Rev. Enferm. UFSM. 2019, v.9, p.1-21 [Citado em 08 março 2021]. Disponível em: https://doi.org/10.5902/2179769232528 